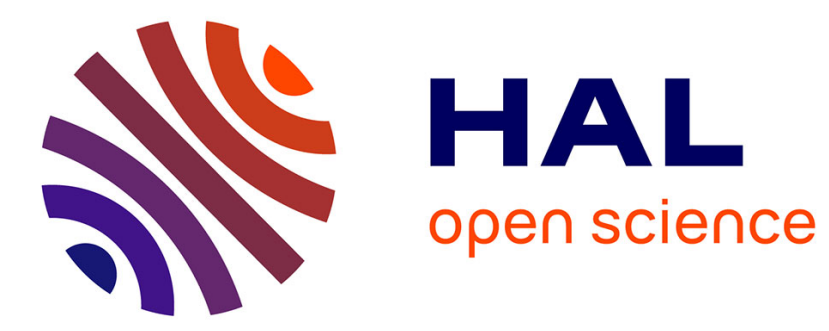

\title{
Effective Human-Robot Collaboration in near symmetry collision scenarios
}

\author{
José Grimaldo da Silva Filho, Anne-Hélène Olivier, Armel Crétual, Julien \\ Pettré, Thierry Fraichard
}

\section{- To cite this version:}

José Grimaldo da Silva Filho, Anne-Hélène Olivier, Armel Crétual, Julien Pettré, Thierry Fraichard. Effective Human-Robot Collaboration in near symmetry collision scenarios. RO-MAN 2019 - 28th IEEE International Conference on Robot \& Human Interactive Communication, Oct 2019, New Dehli, India. pp.1-8. hal-02267705

\section{HAL Id: hal-02267705 \\ https://hal.inria.fr/hal-02267705}

Submitted on 19 Aug 2019

HAL is a multi-disciplinary open access archive for the deposit and dissemination of scientific research documents, whether they are published or not. The documents may come from teaching and research institutions in France or abroad, or from public or private research centers.
L'archive ouverte pluridisciplinaire HAL, est destinée au dépôt et à la diffusion de documents scientifiques de niveau recherche, publiés ou non, émanant des établissements d'enseignement et de recherche français ou étrangers, des laboratoires publics ou privés. 


\title{
Effective Human-Robot Collaboration in near symmetry collision scenarios
}

\author{
Grimaldo Silva ${ }^{1}$, Anne-Hélène Olivier ${ }^{2,3}$, Armel Crétual ${ }^{2,3}$, \\ Julien Pettré ${ }^{3}$ and Thierry Fraichard ${ }^{1}$
}

\begin{abstract}
Recent works in the domain of Human-Robot Motion (HRM) attempted to plan collision avoidance behavior that accounts for cooperation between agents. Cooperative collision avoidance between humans and robots should be conducted under several factors such as speed, heading and also human attention and intention. Based on some of these factors, people decide their crossing order during collision avoidance. However, whenever situations arise in which the choice crossing order is not consistent for people, the robot is forced to account for the possibility that both agents will assume the same role i.e. a decision detrimental to collision avoidance. In our work we evaluate the boundary that separates the decision to avoid collision as first or last crosser. Approximating the uncertainty around this boundary allows our collision avoidance strategy to address this problem based on the insight that the robot should plan its collision avoidance motion in such a way that, even if agents, at first, incorrectly choose the same crossing order, they would be able to unambiguously perceive their crossing order on their following collision avoidance action.
\end{abstract}

\section{INTRODUCTION}

With the increased interest in applications that rely on robots sharing spaces with people, it is important that robots are able to integrate seamlessly into these spaces. A robot being able to determine and replicate the manner in which people avoid a collision with each other could allow people to act naturally around the robot [1], potentially reducing cognitive load for people in the environment [2].

There have been several research works on collision avoidance between people during locomotor task. More specifically, previous works highlighted the collaborative nature of this task and that the relative collision avoidance contribution between people depends on crossing order [3] and that crossing order is determined at the start of the collision avoidance interaction between people [4].

However, in certain situations, the manner in which these collision avoidance contributions should be performed is ambiguous - what we call symmetric collision avoidance scenarios. In these scenarios people are unable to determine their crossing order and are, by consequence, unable to guarantee effective collaboration in terms of complementary

\footnotetext{
*This work is partially supported by the Brazilian National Counsel of Technological and Scientific Development (CNPq) and the LabEx PERSYVAL-Lab (ANR-11-LABX-0025-01) funded by the French program Investissement d'avenir.

${ }^{1}$ Univ. Grenoble Alpes, Inria, CNRS, Grenoble INP, LIG, 38000 Grenoble, France

2 Univ Rennes, M2S, Rennes, France

${ }^{3}$ Univ Rennes, Inria, CNRS, Irisa, Rennes, France

E-mail: jose.jgrimaldo@gmail.com, armel.cretual@univ-rennes2.fr, \{anne-helene.olivier, julien.pettre, thierry.fraichard\}@inria.fr
}

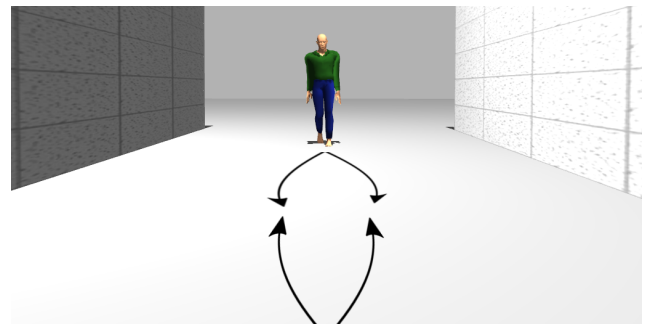

(a) Left or right? A person's decision is ambiguous

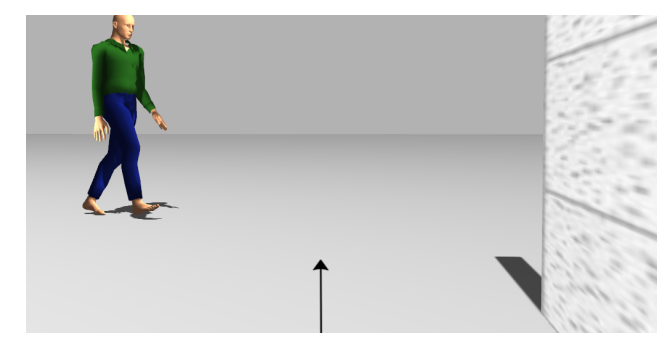

(b) Robot is first crosser but cannot contribute to collision avoidance, should it still pass first or yield?

Fig. 1: Robot's perspective in situations of near symmetry.

motion adaptations to avoid future collision. Some examples of such possible mismatch are shown in Fig. 1, where people may incorrectly choose to pass each other in opposite sides which negatively affects their efforts to avoid collision.

Interpersonal coordination during collision avoidance has been shown to be mainly determined by situational factors such as properties of the environment, relative positions, speed and heading of people rather than their height, gender or personality [4]. However, planning collision avoidance motions solely based on a prediction of crossing order is insufficient to accommodate scenarios of uncertainty as in the absolute worst case of future collision, that happens in symmetric collision scenarios, choosing the most common crossing order is no better than chance because people would choose to cross first or last with equal probability.

In this context, our work focuses on how a robot should move among people, what we call a Human-Robot Motion (HRM) problem. More specifically, we would like to replicate the collaborative nature of motion adaptations between people to avoid collisions during locomotor tasks, particularly whenever the exact manner in which motion adaptations should be performed is ambiguous.

Our objective is to find a collision avoidance motion that 
accounts for potential collaboration to avoid a collision in the best case while also minimizing the chances of crossing order remaining unclear in the worst case, where agents incorrectly assume the same crossing order.

In order to accomplish the above-mentioned goal, we first present a novel measure called Minimum Predicted Distance with Goal Heading (MPDH), in order to analyze data obtained from experiments involving people in situations with different levels of crossing order ambiguity during a collision avoidance task while walking. This measure is used to evaluate whether the motion adaptations of each agent are having an overall positive or negative contribution to collision avoidance. We then establish a relation between the optic variable named derivative of the bearing angle and the uncertainty over crossing order. Using this relation a collision avoidance strategy that minimizes the potential negative impact of crossing order mismatch is then engendered.

\section{BACKGROUND}

People are not regular obstacles, they react to the motion of other agents. Accounting for this reaction allows a robot to minimize its negative impact in a social environment.

The Social Force Model (SFM) can reproduce the manner in which people move and react to other static and dynamic obstacles [5]. This model can be summarized as representing internal motivations of pedestrians through the use of attractive and repulsive forces to elements in the environment.

Using SFM, a robot was able to predict how standing people that are inadvertently blocking its way would move to allow the robot to pass [6]. Similarly, Ferrer and Sanfeliu relied on SFM to predict the reaction of several people to a given robot motion plan so that a trajectory that minimizes the combined reaction of people could be found. [7].

Even though SFM has been extensively used to simulate crowds, it was empirically demonstrated that distance-based repulsive forces have difficulty reproducing individual pedestrian behavior [8].

Another approach, named Reciprocal Velocity Objects (RVO) [9] can also be used to replicate the behavior of crowds [10]. However, RVO was not modelled to naturally reproduce some behaviors of people during collision avoidance, for instance, people were shown to sometimes reverse their crossing order when avoiding collision [4] even though that entails longer time to avoid collision [1].

Recent works used joint trajectories to represent both usual and reversed crossing order of people during collision avoidance [11], [12]. This allowed estimation of possible choices of crossing order for each agent and its associated trajectory. In contrast, our work is focused on near symmetric situations in which crossing order decision is ambiguous which can cause agents to choose the same crossing order. Our collision avoidance approach intends to reduce the negative impact of this ambiguity in the collision avoidance.

\section{CHARACTERIZING A FUTURE COLLISION SCENARIO BETWEEN PEOPLE}

Before presenting our approach for collision avoidance between robots and people, it is necessary to first characterize a future collision scenario. Our focus is on dyadic collision avoidance situations where agents have goal directed trajectories. In order to reproduce behavior of people in these situations, we introduce concepts associated to crossing order determination and a metric of collision avoidance progress.

\section{A. Minimum Predicted Distance}

In order to evaluate the consequences of crossing order uncertainty, a way to numerically represent its impact in the collision avoidance progress is fundamental. In our work, this progress is measured between the start and end of the interaction between people, which we represent as the bounds of the time interval in which people deviate from their desired velocity in order to avoid future collision.

Collision avoidance progress can be measured in terms of a predictive variable named MPD [13]. This variable is presented as the distance where people would meet in the future in case no motion adaptations are performed and is defined as

$$
\begin{aligned}
\operatorname{MPD}(t)=\min _{l=t}^{\infty} \|\left(\boldsymbol{p}_{r}(t)\right. & \left.+\boldsymbol{v}_{r}(t) \cdot(l-t)\right) \\
& -\left(\boldsymbol{p}_{p}(t)+\boldsymbol{v}_{p}(t) \cdot(l-t)\right) \|
\end{aligned}
$$

where $\boldsymbol{p}_{r}(t)$ and $\boldsymbol{p}_{p}(t)$ represent, respectively, the current position of an agent $r$ and $p$ at time $t$. In the same manner, $\boldsymbol{v}_{r}$ and $\boldsymbol{v}_{p}$ represent, respectively, the current velocity of agent $r$ and $p$ and $\|\cdot\|$ denotes the euclidean distance. Thus, whenever people are at a certain distance from each other and MPD is below a certain threshold, people adapt their motion in order avoid collision - increasing the value of MPD. On the other hand, in (near) symmetry situations agents might incorrectly choose the same crossing order which might not increase or even reduce MPD.

\section{B. Derivative of bearing angle in collision avoidance}

The bearing angle denotes the angle between the heading of an agent and another obstacle (dynamic or otherwise). In [14], the derivative of bearing angle was found to correctly predict future collision and also to be a strong indicator of crossing order.

Given this context, let $r$ and $p$ represent a robot and a person respectively. From the local coordinate space of the robot, the bearing angle of $r$ with respect to $p$ is defined as $\alpha_{r, p}(t)=\operatorname{atan} 2(y, x)$ where $x=x_{p}(t)-x_{r}(t)$ and $y=$ $y_{p}(t)-y_{r}(t)$. The derivative of $\alpha_{r, p}(t)$ is defined as

$$
\dot{\alpha}_{r, p}(t)=\frac{y}{x^{2}+y^{2}} \mathrm{~d} x+\frac{x}{x^{2}+y^{2}} \mathrm{~d} y,
$$

where $x_{r}(t)$ and $y_{r}(t)$ represent the $x$ and $y$ position of agent $r$ at time $t$.

The relationship between the derivative of bearing angle and crossing order was explained in terms of the visual stimuli [14]. The first crosser sees the last crosser diverge from the center of its field of view (bearing angle goes away from zero) as the time to collision diminishes, while the last crosser sees the first crosser converging towards the center of 
its field of view (bearing angle approaches zero). These roles were shown in [14] and [3] to affect the manner in which people avoid collisions with each other and were shown to be strongly correlated to the derivative of the bearing angle at the start of collision avoidance.

In order to formalize the decision of an agent based on its crossing order, while also accounting for situations in which crossing order is undefined, such as in headon collision scenarios, the concept of homotopy class is used. This concept was defined in [11], [15] as the side in which agents pass each other. In any given dyadic collision avoidance situation at least one agent has to decide whether they will pass the other on the left or on the right side. Each discrete decision about whether to pass left or right of someone represents a homotopy class decision. In [11], the homotopy class of each distinct trajectory pair was calculated using

$$
\Theta_{r, p}=\int_{t} \dot{\alpha}_{r, p}(t) d t
$$

where $\dot{\alpha}_{r, p}(t)$ represents the derivative of the bearing angle between full trajectories of $r$ and $p$. In this formulation, agents that pass each other on their right side will generate a $\Theta_{r, p}=\pi$ while passing each other on the left side results in $\Theta_{r, p}=-\pi$. This formulation is elegant as its value is independent of the duration of collision avoidance. However, it is important to note that although $\Theta_{r, p}$ correctly associates the choice of homotopy class of two actual (or predicted) trajectories, to complement these results [11] also built a probability distribution that is able to predict the most likely choice of homotopy class for any given person in the environment.

Our hypothesis is that predicting the probability of each distinct homotopy class decision is not sufficient. Understanding the underlying causes and consequences of this ambiguity allows one to explicitly accommodate the possibility of agents choosing actions that, when considered in conjunction with the robot's action, do not contribute to collision avoidance.

The concept of crossing order can then be used as a basis to predict, at any given time $t$, the homotopy class decision of an agent. However, attempting to directly utilize this information to determine collision avoidance motions is insufficient. The reasons for this can be contextualized given a direct representation of such predictor, presented as

$$
\hat{\Theta}_{r, p}(t)= \begin{cases}+\pi \text { or }-\pi, & \text { if } \dot{\alpha}_{r, p}(t)=0 \\ -\pi, & \text { if } \alpha_{r, p}(t) \geq 0 \text { and } \dot{\alpha}_{r, p}(t)>0 \\ -\pi, & \text { if } \alpha_{r, p}(t)<0 \text { and } \dot{\alpha}_{r, p}(t)>0 \\ +\pi, & \text { if } \alpha_{r, p}(t) \geq 0 \text { and } \dot{\alpha}_{r, p}(t)<0 \\ +\pi, & \text { if } \alpha_{r, p}(t)<0 \text { and } \dot{\alpha}_{r, p}(t)<0\end{cases}
$$

which can then be simplified to

$$
\hat{\Theta}_{r, p}(t)= \begin{cases}+\pi \text { or }-\pi, & \text { if } \dot{\alpha}_{r, p}(t)=0 \\ -\pi, & \text { if } \dot{\alpha}_{r, p}(t)>0 \\ +\pi, & \text { if } \dot{\alpha}_{r, p}(t)<0\end{cases}
$$

From this formulation, it is possible to verify that ambiguity could occur in two scenarios: whenever $\alpha_{r, p}(t) \approx 0$ and $\dot{\alpha}_{r}^{p}(t) \approx 0$, that is, a future (near) head-on collision, and in the (near) symmetric cases when $\alpha_{r, p}(t) \not 0$ and $\dot{\alpha}_{r, p}(t) \approx 0$. In both of these scenarios each person is met with a situation where $+\pi$ and $-\pi$ are possible solutions. As a consequence, in the space of solutions of these ambiguous scenarios, denoted as $\hat{\Theta}_{r, p}(t) \times \hat{\Theta}_{p, r}(t)=$ $\{\{\pi, \pi\},\{-\pi,-\pi\},\{\pi,-\pi\},\{-\pi, \pi\}\}$, people may try to pass each other on the same side or may incorrectly try to pass each other in opposite sides, which will negatively affect their effort to avoid future collision e.g. decrease MPD. In both ambiguity scenarios, the uncertainty over crossing order depends on the value of $\dot{\alpha}$, our objective is to predict the uncertainty associated with this boundary, evaluate the potential consequences of this uncertainty in generated motions and, finally, minimize the risk of collision while allowing for effective collaboration.

\section{CROSSING ORDER UNCERTAINTY FROM PEOPLE DURING COLLISION AVOIDANCE}

In this section we evaluate empirical data obtained from collision avoidance situations between people. Our focus is determining the chance that at least one will move in a way that changes their crossing order as predicted by $\dot{\alpha}$.

In our collision avoidance dataset, described in details in [16], we evaluate dyadic interactions between people when avoiding collisions with each other. The experiments were composed of over 450 runs divided about equally into five distinct crossing angles: $30^{\circ}, 60^{\circ}, 90^{\circ}, 120^{\circ}$ and $150^{\circ}$. Several filters were used into the data to remove cases with too much noise resulting in 312 actual experiments for analysis.

The start and end of the interaction are denoted as, respectively, $t_{i}$ and $t_{f}$. As such, our main focus is on the cases where the crossing order changed within the interval $\left[t_{i}, t_{f}\right]$ and whether these changes are associated to the derivative of bearing angle.

\section{A. Behavior of people in scenarios of uncertainty}

In order to directly visualize situations where crossing order is misjudged within our dataset, we modify MPD formulation slightly to obtain a monotonically increasing function whenever crossing order is respected, named Minimum Predicted Distance with Goal Heading (MPDH) and defined as

$$
\begin{aligned}
\operatorname{MPDH}(t)=\min _{l=t}^{\infty} \|\left(\boldsymbol{p}_{r}(t)\right. & \left.+\boldsymbol{v}_{r}^{\mathrm{des}}(t) \cdot(l-t)\right) \\
& -\left(\boldsymbol{p}_{p}(t)+\boldsymbol{v}_{p}^{\mathrm{des}}(t) \cdot(l-t)\right) \|
\end{aligned}
$$

where $\boldsymbol{v}_{r}^{\text {des }}$ is the agent $r$ desired walking velocity at this time if there were no obstacles. This formulation of MPDH is 


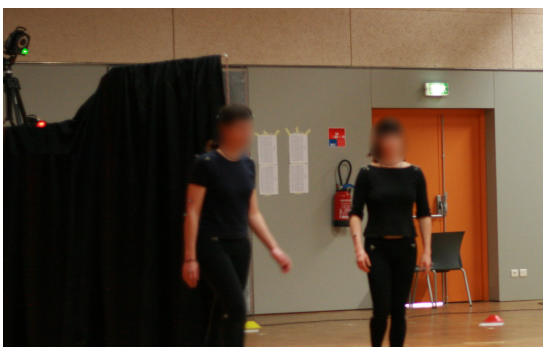

(a) Reconstruction of human motion from data obtained by infrared cameras.

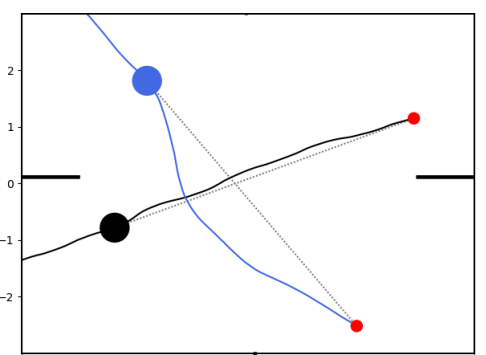

(b) Trajectories in crossing order mismatch situation. First crosser in black.

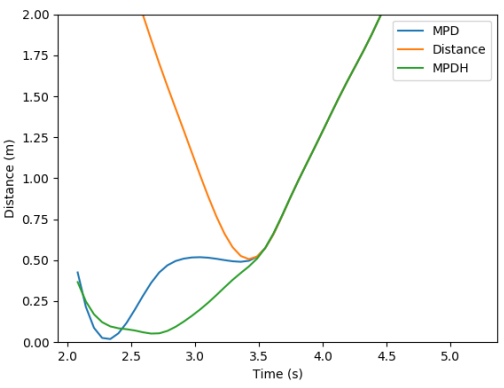

(c) Non-monotonically increasing MPDH.

Fig. 2: Human trajectory comparison when crossing order is misjudged.

the minimum distance considering the hypothetical scenario where the agents have their desired velocity towards the goal. In this sense, it represents the collision potential in terms of its impact on an agent's ability to head towards his goal. As such, whenever crossing order is respected its value increases monotonically during collision avoidance.

This formulation, in contrast with MPD, depends on having the complete trajectories, as in our case the goal of each agent and their desired speed (before collision avoidance) can be easily determined from their trajectories. This measure was used in the result analysis section (see Sec. VI-B) and to evaluate collision avoidance trajectories in our dataset.

As shown in Fig. 2, MPDH was used to determine the impact of crossing order changes in the progress of the collision avoidance.

\section{B. Determining uncertainty boundaries}

Our approach relies on predicting the behavior of agents when they are avoiding each other even in case of ambiguous crossing order. In Fig. 3 we depict some possible trajectories for reactive collision avoidance systems in case crossing order mismatches happen. To complement these results, in Sec. VI, we compare this expected result with our approach.

The derivative of the bearing angle $\dot{\alpha}$ is a strong predictor of crossing order, as shown in [14]. However, the role of each agent in a collision avoidance situation is not always clear. In this section, our focus is on first establishing a relationship between $\dot{\alpha}$ and the certainty in crossing order determination using our dataset. Our objective is to find what is the chance that in the interval between $\left[t_{i}, t_{f}\right]$ the predicted crossing order changes, that is, the probability of $\hat{\Theta}\left(t_{i}\right) \neq \hat{\Theta}(t)$ for some $t \in\left[t_{i}, t_{f}\right]$.

The results, fitted to a sigmoid $S(z)=\frac{1}{1+e^{-a(z-b)}}$ with parameters $a=39.936914$ and $b=-0.000037$, are shown at Fig. 4 indicate that, at $t_{i}$, if $\dot{\alpha}$ approaches zero the likelihood of the crossing order changing within a given collision situation increases. As an extra measure of certainty, to guarantee this is not caused by reconstruction error in the position or heading, we manually evaluated the situations in which crossing order changed and verified that in most cases collision avoidance actions for agents, in terms of speed and heading changes or lack thereof, when observed in conjunction, were initially detrimental to collision avoidance.

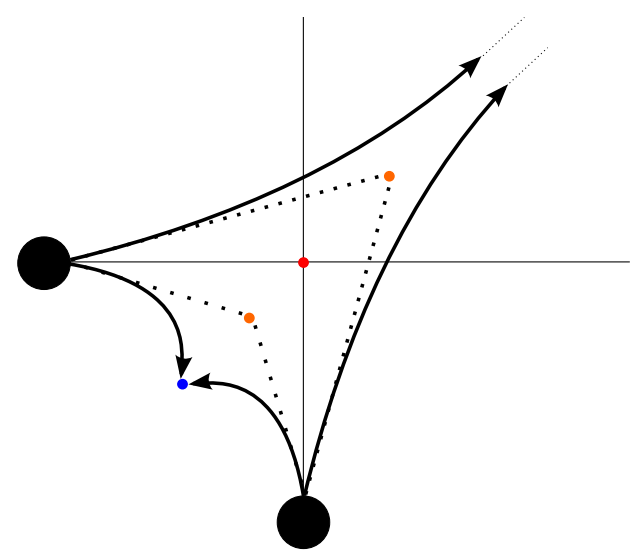

Fig. 3: Reactive collision avoidance system whenever agents incorrectly choose the same crossing order. Point in red is the collision in case of no change in trajectory. Collision point in orange happens if the agents plan collision avoidance only once. Collision in the blue point when both agents continuously choose to cross last. Whenever agents continuously believe they cross first they reach a stable forward motion without ever colliding with each other or reaching their goal.

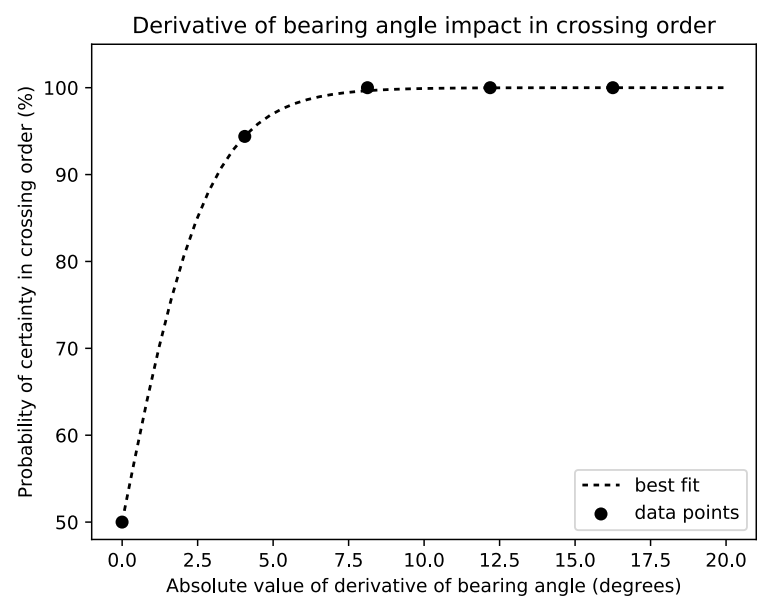

Fig. 4: Evaluation of 312 collision avoidance scenarios, divided into five partitions. Each partition represents the probability that the predicted homotopy class does not change at any point in the interval $\left[t_{i}, t_{f}\right]$. 


\section{Establishing crossing order in symmetric scenarios}

In our work, we attempt to establish effective collision avoidance between agents in symmetric scenarios in up to $n$ decisions, with agents taking two decisions per second. Effective collision avoidance means that both agents choose different crossing orders to avoid each other.

To that end, it is important to calculate the probability of correct collaboration $\mathcal{D}$ that would allow for $\Lambda$ confidence in resolving crossing order after $n$ consecutive attempts. In our work we define $95 \%$ as an acceptable $\Lambda$ value. To find the value of $\mathcal{D}$ we equate the chance of $n$ consecutive failures with $1-\Lambda$, as

$$
1-\Lambda=[1-\mathcal{D}]^{n}
$$

which yields

$$
\mathcal{D}=1-\sqrt[n]{1-\Lambda}
$$

This means that, at each time step, the agent should attempt to guarantee that the chance of resolving crossing order is at least $\mathcal{D}$. As seen in Sec. IV-B, crossing order certainty can be calculated using $\mathcal{S}\left(\dot{\alpha}_{r, p}(t)\right)$, as such, at this time step in case $\mathcal{D} \leq \mathcal{S}\left(\dot{\alpha}_{r, p}(t)\right)$ the optimal collision avoidance action would generally suffice. However, our approach also anticipates situations with ambiguous crossing order by controlling the derivative of the bearing angle in an attempt to guarantee that, in the next time step, the chance of collaboration will be, in average, enough to achieve the desired confidence over $n$ steps.

Consider that the robot $r$ decides to avoid the collision with an agent $p$ by changing its velocity to $\boldsymbol{v}_{r}^{\text {new }}$, this decision is made based on the current velocity of the other agent, which we denote as $\boldsymbol{v}_{p}(t)$. This motion ${ }^{1}$, in case of no change in the behavior of $p$, would yield $\dot{\alpha}_{r, p}^{\text {new }}$. Similarly, agent $p$ would use a similar logic to obtain a possibly distinct $\dot{\alpha}_{p, r}^{\text {new }}$. Based on Eq. 2, the combined effect of these individual velocity changes on the instantaneous value of the derivative of the bearing angle can be calculated using $\dot{\alpha}_{r, p}^{\text {new }}+\dot{\alpha}_{p, r}^{\text {new }}$.

Whenever agents incorrectly choose the same crossing order $\dot{\alpha}_{r, p}^{\text {new }}$ and $\dot{\alpha}_{p, r}^{\text {new }}$ will have distinct signs i.e. one will be positive and the other will be negative. Our objective is to calculate the probability $\mathcal{P}$ that $\left|\dot{\alpha}_{r, p}^{\text {new }}+\dot{\alpha}_{p, r}^{\text {new }}\right|$ in the next decision step will be equal or larger than a given threshold $\kappa$. This would guarantee a certain confidence that even if agents at first incorrectly choose the same crossing order the crossing order would not remain ambiguous at the next decision step.

The desired value of $\mathcal{P}(z)$ where $z=\dot{\alpha}_{r, p}(t)$ at current time step can be calculated by equating the probability of two incorrect collaborations at the current and subsequent decision step to the current $1-\mathcal{S}\left(\dot{\alpha}_{r, p}(t)\right)$ and the subsequent one where $\left|\dot{\alpha}_{r, p}^{\text {new }}+\dot{\alpha}_{p, r}^{\text {new }}\right|$ was not larger than $\kappa$, with

\footnotetext{
${ }^{1}$ Do note that although $\dot{\alpha}_{r, p}(t)=\dot{\alpha}_{p, r}(t)$, the values of $\dot{\alpha}_{r, p}^{\text {new }}$ and $\dot{\alpha}_{p, r}^{\text {new }}$ are not necessarily equal as in this case we are evaluating the new velocity of one agent against the current velocity of the other agent (and vice-versa).
}
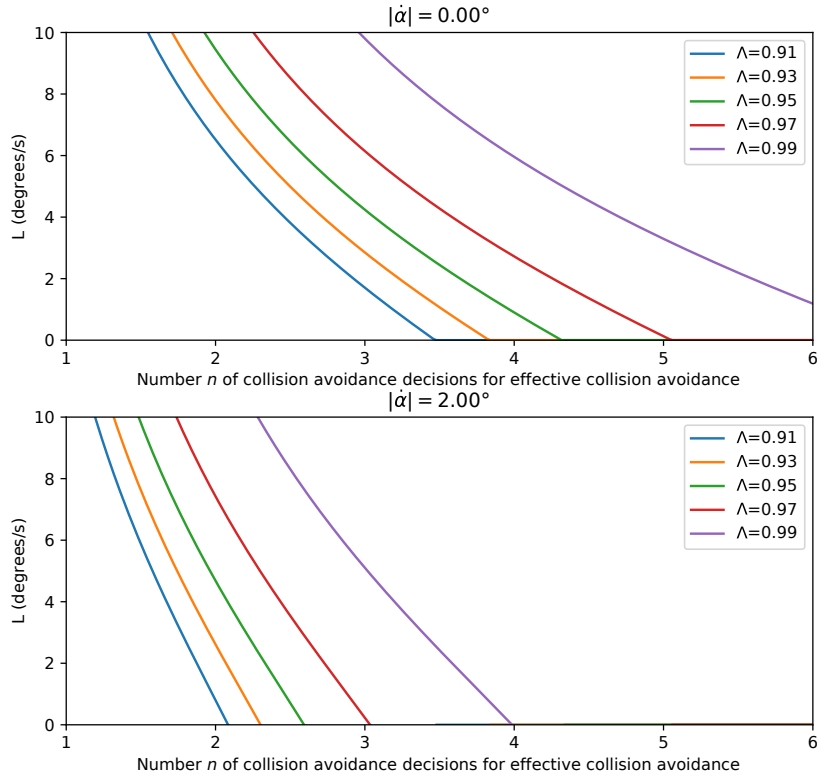

Fig. 5: Relationship between the current $\dot{\alpha}_{r, p}(t)$, the desired number of decisions $n$ to start effective collaboration and $\mathcal{L}$.

$$
\mathcal{P}(z)=1-\frac{(1-\mathcal{D}) \cdot(1-\mathcal{D})}{1-\mathcal{S}(z)}
$$

Our final steps are then to determine the threshold value $\kappa$ and to determine the manner in which one can guarantee $\left|\dot{\alpha}_{r, p}^{\text {new }}+\dot{\alpha}_{p, r}^{\text {new }}\right| \geq \kappa$ with average probability $\mathcal{P}(z)$.

To this end, let $\mathcal{X}$ be a random variable uniformly distributed over the interval $[0, \mathcal{L}]$. From this random variable we select two points $\mathcal{X}_{1}$ and $\mathcal{X}_{2}$. The distance between these points, denoted as $Y=\left|\mathcal{X}_{1}-\mathcal{X}_{2}\right|$, has an average value of $M=\mathbb{E}[Y]=\frac{L}{3}$ (see appendix for details) where $\mathbb{E}[\cdot]$ is the expected value. Due to the locally linear shape of its function, we assume that a sample from $Y$ that is $m$ units away from the mean approximately respects the equality $\mathcal{P}(M)=\frac{\mathcal{P}(M-m)+\mathcal{P}(M+m)}{2}$, this would mean that $\mathcal{P}(\mathbb{E}[Y])=\mathbb{E}[\mathcal{P}(Y)]$. This approximation allows us to establish that with $M=\kappa$ we can finally determine the value of $\kappa$ using the inverse of the sigmoid function (logit) presented in Sec. IV-B, denoted as $\mathcal{S}^{-1}(w)=b+\frac{1}{a} \log \left(\frac{w}{1-w}\right)$, as a function of the desired probability of collaboration in the next time step with $\kappa=\mathcal{S}^{-1}(\mathcal{P}(z))$.

With these elements we can choose motions that respect the relation $\left|\dot{\alpha}_{r, p}^{\text {new }}+\dot{\alpha}_{p, r}^{\text {new }}\right| \geq \kappa$ with average probability equal to $\mathcal{P}(z)$ by sampling $\dot{\alpha}_{r, p}^{\text {new }}$ from a uniform distribution with interval length $\mathcal{L}=3 \kappa$.

The relationship between $n, \dot{\alpha}_{r, p}(t)$ and $\mathcal{L}$ are shown in Fig. 5. As $n$ increases the size of $\mathcal{L}$ decreases until it it reaches zero which signifies that the agent is guessing crossing order based on just its current $\dot{\alpha}_{r, p}(t)$. This means that whenever $\dot{\alpha}_{r, p}(t)=0$ both agents would be choosing crossing order with odds no better than chance unless $\mathcal{L}>0$. 

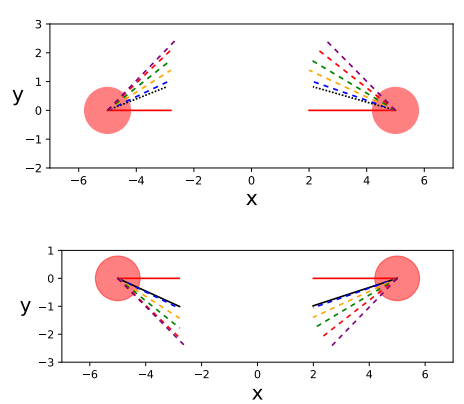

(a) Head-on future collision with different initial speed. Crossing order is not defined.

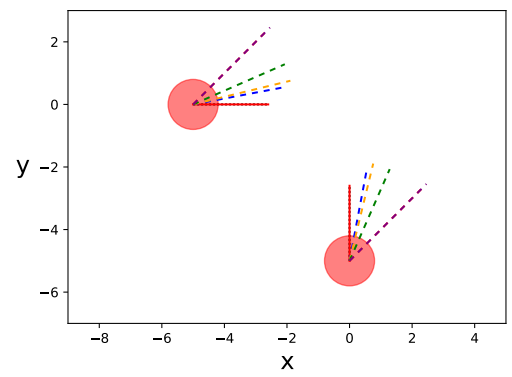

(b) Both assume first crosser role. Future collision with $90^{\circ}$ crossing angle. Constrained by maximum velocity.

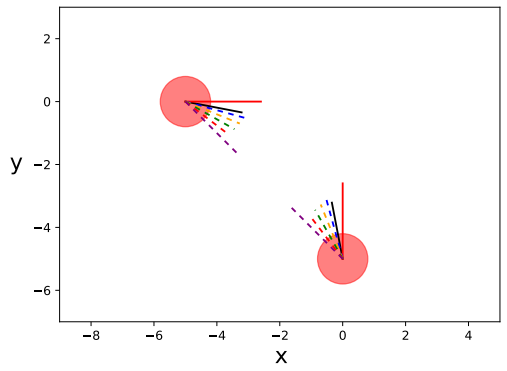

(c) Both assume last crosser role. Future collision with $90^{\circ}$ crossing angle. Less constrained by minimum velocity.

Fig. 6: Samples of collision avoidance motions with misjudged crossing order. Each color in a dashed line indicates a specific choice of derivative of bearing angle. Solid red and black lines indicate, respectively, initial motion and motion with $\dot{\alpha}_{r, p}^{-}$.

\section{Discussion}

Our approach to tackle near symmetry scenarios during collision avoidance is using communication through motion. The agent samples from a uniform distribution of size $\mathcal{L}$ the value $\dot{\alpha}_{r, p}^{\text {new }}$ in relation to the person's current perceived motion to have $\Lambda$ confidence that effective collision avoidance will start in $n$ time steps. Given our results, both $n=2$ and $n=3$ were found achievable for a robot. However, depending on the crossing order and time to collision $n=2$ sometimes imposed a confidence smaller than $\Lambda$.

Another potential limitation is a consequence of a short time to collision, which would impose limits on the range of motions that can still avoid collision and by consequence limit the range of derivative of bearing angles that can be explored. Given this situation and considering that people generally need at least two steps for motion adaptation [17], we chose to focus our approach in situations where time to collision is above one second.

\section{COLLISION AVOIDANCE UNDER NEAR-SYMMETRY}

In the context of dyadic collision avoidance, our premise is that each individual agent will do its best to avoid future collision while preserving crossing order. The range of actions an agent can perform is limited by their maximum linear and angular velocity. Considering these constraints, each individual agent will attempt to minimize the change in their desired velocity $\boldsymbol{v}_{r}^{\text {des }}(t)$ that can avoid the future collision. Each agent, at first, will do the best they can to avoid a future collision, this can be represented as

$$
\boldsymbol{v}_{r}^{*}=\underset{\boldsymbol{v} \in \mathcal{F}_{r}^{p}}{\arg \min }\left\|\boldsymbol{v}-\boldsymbol{v}_{r}^{\mathrm{des}}(t)\right\|
$$

where $\mathcal{F}_{r}^{p}$ is the set of velocities for $r$ where MPD of $r$ with respect to $p$ is larger than the threshold for collision and also respects crossing order i.e. attempts to guarantee that $\hat{\Theta}_{r}^{p}\left(t_{i}\right)=\hat{\Theta}_{r}^{p}(t)$ for all $t \in\left[t_{i}, t_{f}\right]$.

However, as was shown in Sec. IV, crossing order can be misjudged which can have a detrimental impact on collision avoidance. As such, first we define an additional constraint on $\boldsymbol{v}$ based on the $\dot{\alpha}_{r, p}^{\text {new }}$ it would generate, represented as

$$
\dot{\alpha}_{r, p}^{\text {new }}=R\left(\dot{\alpha}_{r, p}^{-}, \dot{\alpha}_{r, p}^{+}\right)
$$

where $R\left(\dot{\alpha}_{r, p}^{-}, \dot{\alpha}_{r, p}^{+}\right)$is a random value in the interval defined by $\dot{\alpha}_{r, p}^{-}$and $\dot{\alpha}_{r, p}^{+}$, which represent, respectively, the lower and upper bound in possible values of the derivative of bearing angle for $r$ with respect to $p$ that can avoid collision in a given crossing order. To generate collision avoidance velocities that respect crossing order we use an optimization approach named Sequential Least SQuares Programming (SLSQP) [18] as it can handle any combination of bounds, equality and inequality constraints.

To guarantee the desired confidence, it is necessary that $\mathcal{L} \leq\left|\dot{\alpha}_{r, p}^{+}-\dot{\alpha}_{r, p}^{-}\right|$. It is only necessary to select a subset of the interval $\left|\dot{\alpha}_{r, p}^{+}-\dot{\alpha}_{r, p}^{-}\right|$with $\mathcal{L}$ length. Examples of possible collision avoidance motions with a specific $\dot{\alpha}_{r, p}^{\text {new }}$, from a discrete subset of values within an interval of size $\mathcal{L}$, are shown in Fig. 6.

Given perceived safety and kinematic constraints, it is possible that $\mathcal{L} \geq\left|\dot{\alpha}_{r, p}^{+}-\dot{\alpha}_{r, p}^{-}\right|$, in these cases the agent would still attempt to resolve the collision with reduced confidence. However, when time to collision is below one timestep with $\mathcal{L} \geq\left|\dot{\alpha}_{r, p}^{+}-\dot{\alpha}_{r, p}^{-}\right|$the agent yields as it does not have the required confidence for human-like collaboration. These situations are more frequent whenever agents have a time to collision below one second.

In situations where crossing order has already a confidence value higher than $\Lambda$, which means that $\mathcal{L}=0$, it is possible to preserve crossing order even when one agent cannot contribute to avoid a collision (or its contribution would be insufficient). For instance, as shown in Fig. 1b, in many situations the first crosser is unable to accelerate further or change heading in a manner that preserves crossing order and they are thus unable to contribute to collision avoidance. Nonetheless, in scenarios with clear crossing order the first crosser would be able to rely on the last crosser to avoid collision even without any cooperation.

In any similar case, it is fundamental that crossing order is unambiguous as otherwise the last crosser could incorrectly 


\begin{tabular}{clcccc}
\hline $\begin{array}{l}\text { Crossing } \\
\text { angle }\end{array}$ & $\begin{array}{l}\text { Time to } \\
\text { collision }\end{array}$ & $\left|\dot{\alpha}_{r, p}\left(t_{i}\right)\right|$ & $n$ & $\mathcal{L}$ & $\left|\dot{\alpha}_{r, p}^{-}-\dot{\alpha}_{r, p}^{+}\right|$ \\
\hline $30^{\circ}$ & $2.89 \mathrm{~s}$ & $1.40^{\circ}$ & 3 & $0.06^{\circ}$ & $\mathbf{3 . 0 0}^{\circ}$ \\
$30^{\circ}$ & $2.53 \mathrm{~s}$ & $0.47^{\circ}$ & 3 & $3.15^{\circ}$ & $2.50^{\circ}$ \\
$30^{\circ}$ & $2.47 \mathrm{~s}$ & $1.03^{\circ}$ & 3 & $1.48^{\circ}$ & $\mathbf{2 . 5 0}^{\circ}$ \\
$30^{\circ}$ & $2.11 \mathrm{~s}$ & $0.75^{\circ}$ & 3 & $2.38^{\circ}$ & $\mathbf{3 . 0 0}^{\circ}$ \\
$30^{\circ}$ & $2.05 \mathrm{~s}$ & $2.22^{\circ}$ & 3 & $0.00^{\circ}$ & $\mathbf{1 . 2 5}^{\circ}$ \\
$30^{\circ}$ & $1.64 \mathrm{~s}$ & $1.13^{\circ}$ & 3 & $1.13^{\circ}$ & $0.50^{\circ}$ \\
\hline $120^{\circ}$ & $2.58 \mathrm{~s}$ & $1.20^{\circ}$ & 2 & $6.95^{\circ}$ & $\mathbf{7 . 5 0}^{\circ}$ \\
$120^{\circ}$ & $2.52 \mathrm{~s}$ & $0.77^{\circ}$ & 2 & $7.96^{\circ}$ & $7^{\circ} .25^{\circ}$ \\
$120^{\circ}$ & $2.51 \mathrm{~s}$ & $10.09^{\circ}$ & 2 & $0.00^{\circ}$ & $\mathbf{1 0 . 7 5}^{\circ}$ \\
$120^{\circ}$ & $2.17 \mathrm{~s}$ & $9.01^{\circ}$ & 2 & $0.00^{\circ}$ & $\mathbf{1 1 . 2 5}^{\circ}$ \\
$120^{\circ}$ & $1.75 \mathrm{~s}$ & $2.65^{\circ}$ & 2 & $2.33^{\circ}$ & $\mathbf{8 . 0 0}^{\circ}$ \\
$120^{\circ}$ & $1.69 \mathrm{~s}$ & $1.64^{\circ}$ & 2 & $5.77^{\circ}$ & $\mathbf{6 . 0 0}^{\circ}$ \\
\hline
\end{tabular}

TABLE I: Evaluation of six random variations of two near symmetrical collision scenarios with specific crossing orders. Positive results, where the value of $\left|\dot{\alpha}_{r, p}^{-}-\dot{\alpha}_{r, p}^{+}\right|$is larger than $\mathcal{L}$, are marked in bold. As crossing order is chosen randomly it can affect the length of $\left|\dot{\alpha}_{r, p}^{-}-\dot{\alpha}_{r, p}^{+}\right|$in similar scenarios (crossing last usually allows for larger interval). In the case with $120^{\circ}$ crossing angle a smaller $n$ was used.

perceive himself as first crosser. This would ultimately result in collision if both agents are unable to contribute to collision avoidance as first crosser.

\section{SIMULATED EXPERIMENTS}

Evaluating whether agents can avoid collision in near symmetry scenarios requires analysis of data from the agents' motion in situations with ambiguous crossing order. To that end, several simulated experiments analysing different aspects of our approach. are described in this section.

In our experiments, a holonomic motion model was chosen for the robot but the change in heading direction $\theta_{r}$ between decision steps is bounded as $-\frac{\pi}{2}<\dot{\theta}_{r}<\frac{\pi}{2}$ ( $\mathrm{rad} / \mathrm{s}$ ) during collision avoidance to allow for more predictable motions. Similarly, the maximum speed of both agents was set as $v_{r}^{\max }=v_{p}^{\max }=1.7(\mathrm{~m} / \mathrm{s})$ and initial speed as $s_{r}^{\text {initial }}=$ $s_{p}^{\text {initial }}=1.2(\mathrm{~m} / \mathrm{s})$.

Finally, to avoid sudden turns during collision avoidance, which would negatively affect motion predictability for people, robot motions are smoothed using cubic splines.

\section{A. Impact of linear and angular constraints on near- symmetry mitigation}

Although we establish the condition $\mathcal{L} \leq\left[\dot{\alpha}_{r, p}^{-}, \dot{\alpha}_{r, p}^{+}\right]$for $\Lambda$ confidence in effective collision avoidance, it is important to evaluate when, in practice, the robot can obtain a reasonable length of the interval $\left[\dot{\alpha}_{r, p}^{-}, \dot{\alpha}_{r, p}^{+}\right]$when the constraints in both $v_{r}^{\max }$ and $\dot{\theta}_{r}$ are taken into account.

To that end, several random variations of collision avoidance scenarios were simulated over two crossing orders. The results, calculated considering $\Lambda=95 \%$ and showcased in Table I, indicate that, when time to collision is small, the length of the interval $\left[\dot{\alpha}_{r, p}^{-}, \dot{\alpha}_{r, p}^{+}\right]$also decreases. In these situations, collision avoidance requires sharper changes in speed and heading, this means that a smaller range of motions that are still able to avoid collision can be achieved.
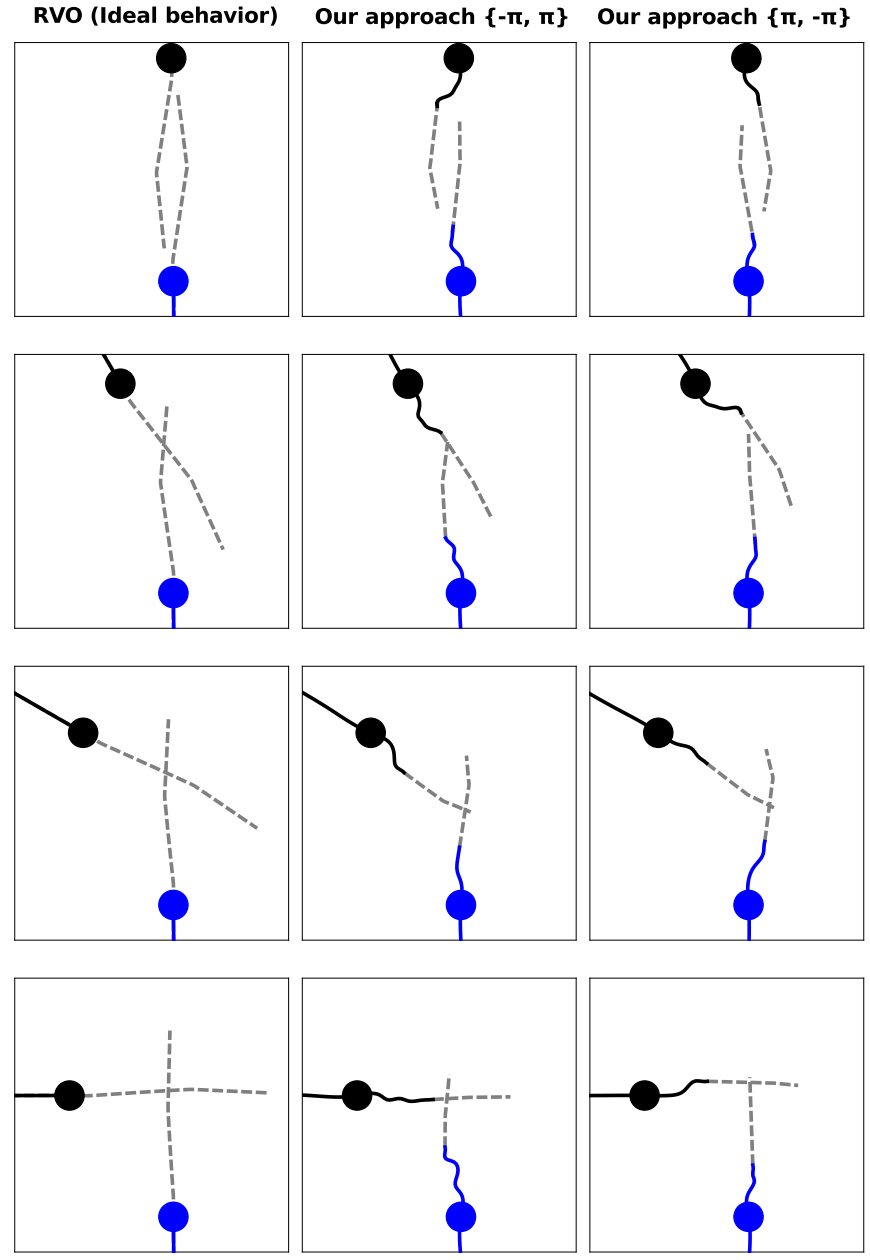

Fig. 7: Comparison of ideal scenarios where people always respect crossing order to our approach where ineffective collaboration is possible. Our collision avoidance approach mitigated the negative consequences of such event. Continuous lines indicate ambiguous crossing order while dashed gray lines indicate crossing order is no longer ambiguous.

In our analysis it was also shown that in smaller crossing angles three decisions can be necessary to achieve confidence larger than at least $90 \%$. In contrast, collision scenarios with crossing angle of $120^{\circ}$ allowed us to utilize up to $n=2$ while maintaining $\Lambda$ confidence, even though the situations for both crossing angles were generated with the same random process, this indicates that smaller changes in heading and speed generated larger changes in the derivative of the bearing angle when crossing angle was higher.

\section{B. Collision avoidance trajectories under near symmetry}

In order to assess whether generated collision avoidance motions properly mitigate the potential negative impact of ambiguous crossing order, a dozen collision avoidance scenarios where generated for each of the four tested crossing angles, these are: $0^{\circ}, 30^{\circ}, 60^{\circ}$ and $90^{\circ}$.

In order to establish a comparison baseline with our approach, the collision avoidance of RVO [9] was chosen due to its extensive use in the literature. As can be seen in 

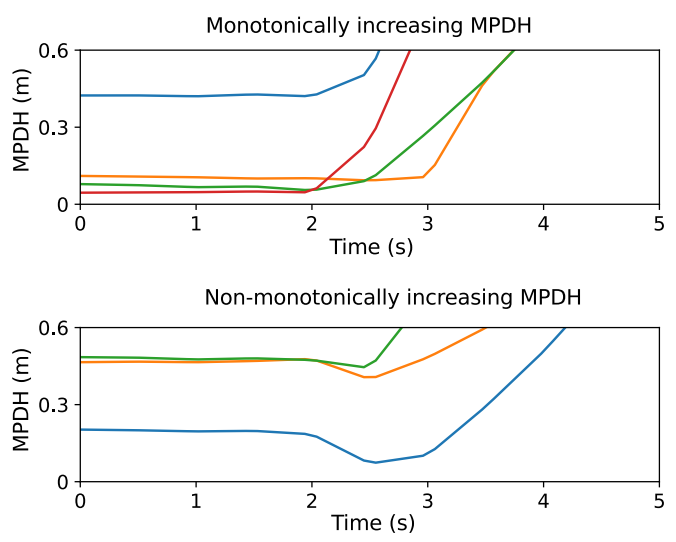

Fig. 8: Several examples of MPDH where agents misjudge crossing order. In the upper plot, the MPDH increased less efficiently during the period where crossing order is misjudged, in the lower plot crossing order being misjudged negatively affected MPDH (its value decreases). Each color represents a distinct collision avoidance situation.

Fig. 7, RVO is only able to reproduce collision avoidance motions where crossing order is respected. Our approach accounts for the possibility that agents misjudge crossing order in near symmetry scenarios.

The negative impact of ineffective collaboration in both agents ability to head towards their goal can be visualized with MPDH. In Fig. 8, an incorrect choice of crossing order can cause MPDH to increase less efficiently or even decrease i.e. negatively affecting their efforts to avoid collision.

In most cases, effective collaboration started after at most three decisions. In under 7\% of the evaluated cases, the nearsymmetry situation was not solved and both agents stopped when time to collision was below one second.

\section{CONCLUSIONS}

This work tackles dyadic collision avoidance situations between a robot and a person when the choice of crossing order for effective collaboration during collision avoidance is unclear. Approximating the uncertainty around this choice allowed our approach to choose a collision avoidance motion in such a way that, even if agents initially chose to cross each other on different sides, the robot and the person were generally able to perceive the correct homotopy class decision on their next decision step.

As future work, an interesting direction would be to run experiments in a virtual reality environment with a robot and a person controlling an agent. This approach avoids the risk of actual collision between robot and person. Moreover, assessing the impact of personal factors, such as cultural norms [19], can be an interesting direction.

\section{APPENDIX}

In this section we provide a justification ${ }^{2}$ of why given two random variables $\mathcal{X}_{1}$ and $\mathcal{X}_{2}$ that are uniformly distributed

\footnotetext{
${ }^{2}$ Empirical validation code is available at https://github.com/ jgrimaldo/demonstrations
}

over the interval $[0, \mathcal{L}]$ their distance $Y=\left|X_{1}-X_{2}\right|$ will be in average $\mathbb{E}[Y]=\frac{\mathcal{L}}{3}$.

Suppose that given $\mathcal{X}_{1}$ and $\mathcal{X}_{2}$ we also choose a third random variable $\mathcal{X}_{3}$, also uniformly sampled from an interval of length $\mathcal{L}$. The value of $\mathcal{X}_{3}$ will be between $\mathcal{X}_{1}$ and $\mathcal{X}_{2}$, in average, $1 / 3$ of the time as they are equiprobable. This means that the distance between $\mathcal{X}_{1}$ and $\mathcal{X}_{2}$ needs to be, in average, a third of the length of the interval and as such can be calculated simply as $\mathbb{E}[Y]=\frac{\mathcal{L}}{3}$.

\section{References}

[1] C. Vassallo, A.-H. Olivier, P. Souères, A. Crétual, O. Stasse, and J. Pettré, "How do walkers behave when crossing the way of a mobile robot that replicates human interaction rules?" Gait \& posture, vol. 60, pp. 188-193, 2018.

[2] D. Carton, W. Olszowy, and D. Wollherr, "Measuring the effectiveness of readability for mobile robot locomotion," Int. J. of Social Robotics, 2016.

[3] A. Olivier, A. Marin, A. Crétual, A. Berthoz, and J. Pettré, "Collision avoidance between two walkers: Role-dependent strategies," Gait and Posture, vol. 38, no. 4, 2013.

[4] A. Knorr, L. Willacker, J. Hermsdörfer, S. Glasauer, and M. Krüger, "Influence of person-and situation-specific characteristics on collision avoidance behavior in human locomotion." Int. J. of experimental psychology: human perception and performance, vol. 42, no. 9, p. 1332, 2016

[5] D. Helbing and P. Molnár, "Social force model for pedestrian dynamics," Physical Review E, vol. 51, no. 5, 1995.

[6] A. Cosgun, E. Sisbot, and H. Christensen, "Anticipatory robot path planning in human environments," in Robot and Human Interactive Communication, New York (UM), Aug. 2016.

[7] G. Ferrer and A. Sanfeliu, "Proactive kinodynamic planning using the extended social force model and human motion prediction in urban environments," in IEEE Int. Conf. on Intelligent Robots and Systems (IROS), Chicago (UM), Sept. 2014.

[8] B. Steffen, "A modification of the social force model by foresight," in Pedestrian and Evacuation Dynamics. Springer, 2010.

[9] J. van den Berg, S. Guy, M. Lin, and D. Manocha, Reciprocal n-Body Collision Avoidance. Berlin, Heidelberg: Springer, 2011.

[10] A. Bera and D. Manocha, "Realtime multilevel crowd tracking using reciprocal velocity obstacles," in Int. Conf. on Pattern Recognition (ICPR). IEEE, 2014.

[11] M. Kuderer, H. Kretzschmar, and W. Burgard, "Teaching mobile robots to cooperatively navigate in populated environments," in Int. Conf. in Intelligent Robots and Systems (IROS). IEEE, 2013.

[12] H. Kretzschmar, M. Spies, C. Sprunk, and W. Burgard, "Socially compliant mobile robot navigation via inverse reinforcement learning," Int. J. of Robotics Research, vol. 35, no. 11, pp. 1289-1307, 2016.

[13] A. Olivier, A. Marin, A. Crétual, and J. Pettré, "Minimal predicted distance: A common metric for collision avoidance during pairwise interactions between walkers," Gait and Posture, vol. 36, no. 3, pp. 399-404, 2012.

[14] J. Cutting, P. Vishton, and P. Braren, "How we avoid collisions with stationary and moving obstacles," American Psychological Association, vol. 102, no. 4, 1995.

[15] M. Kuderer, H. Kretzschmar, C. Sprunk, and W. Burgard, "Featurebased prediction of trajectories for socially compliant navigation." in Robotics: science and systems, 2012.

[16] G. Silva, A.-H. Olivier, A. Cretual, J. Pettre, and T. Fraichard, "Human inspired effort distribution during collision avoidance in human-robot motion," in ROMAN. IEEE, 2018.

[17] A. Patla, "Understanding the roles of vision in the control of human locomotion," Gait \& posture, vol. 5, no. 1, pp. 54-69, 1997.

[18] D. Kraft, "Algorithm 733: TOMP - fortran modules for optimal control calculations," ACM Trans. Math. Softw., vol. 20, no. 3, pp. 262-281, 1994.

[19] U. Chattaraj, A. Seyfried, and P. Chakroborty, "Comparison of pedestrian fundamental diagram across cultures," Advances in complex systems, vol. 12, no. 03, pp. 393-405, 2009. 